Original Research

\title{
Clinical relevance of oncogenic driver mutations identified in endometrial carcinoma
}

\author{
Takafumi Watanabe $^{\mathrm{a}, *}$, Hideaki Nanamiya ${ }^{\mathrm{b}}$, Manabu Kojima ${ }^{\mathrm{a}}$, Shinji Nomura ${ }^{\mathrm{a}}$, \\ Shigenori Furukawa ${ }^{a}$, Shu Soeda ${ }^{a}$, Daisuke Tanaka ${ }^{b}$, Takao Isogai $^{\mathrm{b}}$, Jun-ichi Imai ${ }^{\mathrm{b}}$, \\ Shinya Watanabe ${ }^{\mathrm{b}}$, Keiya Fujimori ${ }^{\text {a }}$ \\ ${ }^{a}$ Department of Obstetrics and Gynecology, Fukushima Medical University, Fukushima 960-1295, Japan \\ b Translational Research Center, Fukushima Medical University, Fukushima 960-1295, Japan
}

\section{A R T I C L E I N F O}

\section{Keywords:}

Endometrial carcinoma

Somatic mutations

Clinical molecular genetics

Prognostic biomarker

\begin{abstract}
A B S T R A C T
Purpose: Endometrial carcinoma (EC) is a clinically heterogeneous disease characterized by a number of different histological subtypes, and its heterogeneity may be involved in the accumulation of multiple genetic alterations. The aim of this work was to investigate the comprehensive mutational profile of EC tumors, and examine the associations between somatic mutations and clinicopathological features or survival in EC patients.

Methods: A total of 100 surgical tumors were obtained from EC patients who had previously undergone surgery. Genomic DNA samples extracted from fresh-frozen tissues were analyzed using the Ion AmpliSeq Cancer Hotspot Panel v2 Kit, covering 50 tumor-related genes.

Results: Validated mutations were detected in 91 of the 100 tumors (91\%) and identified in eight of the most frequently mutated genes, namely PTEN (57\%), PIK3CA (51\%), TP53 (30\%), KRAS (23\%), CTNNB1 (21\%), FBFR2 (13\%), FBXW7(10\%) and RB1 (9\%). PTEN mutations were found to associated with young age $(<60)$, early-stage, endometrioid histology, non-recurrence and better overall survival (OS). CTNNB1 mutations were associated with young age, endometrioid histology and better OS. On the other hands, TP53 mutations were associated with late-stage, non-endometrioid histology, high-grade, recurrence and worse OS. FBWX7 mutations were associated with late-stage, vascular invasion and lymph node metastasis. FGFR2 mutations correlated with deep ( $\geq 1 / 2)$ myometrial invasion.

Conclusion: Our comprehensive mutational profile will be useful for understanding and evaluating the molecular characteristics of EC tumors, and may lead to the establishment of novel treatment strategies that improve the survival of patients with EC in the future.
\end{abstract}

\section{Introduction}

Endometrial cancer (EC) is the most frequently-reported malignancy of the female genital tract, and the seventh most commonly occurring cancer in women worldwide, as of 2018 [1]. EC often produces symptoms, such as postmenopausal or abnormal genital bleeding, at a relatively early-stage, so the disease is generally diagnosed early. Although more than $75 \%$ of EC patients are stage I at the time of diagnosis, and the 5 -year overall survival (OS) rate for women with early-stage EC exceeds $80 \%, 10-15 \%$ of all patients experience recurrence $[1,2]$. Stage, histological type, tumor grade, myometrial invasion (MI), vascular invasion (VI) and lymph node metastasis (LNM) at the time of treatment are defined as independent prognostic factors in patients with EC by the International Federation of Obstetrics and Gynecology (FIGO) [3]. However, to explain EC heterogeneity with these clinicopathological factors alone is impossible [4]. Therefore, identification of new predictive markers for a high-risk phenotype would be very useful for selection of the most efficient therapies as well as for the development of novel treatment modalities.

Recently, comprehensive molecular profiling of cancer using nextgeneration sequencing (NGS) approaches has been increasingly used in oncology for diagnostic and therapeutic management decisions. Cancerspecific mutations, including single-nucleotide alterations and small insertions or deletions, are known to affect key driver genes early during tumorigenesis [5]. There are two types of EC: Type I EC (80-90\%) characterized by an excess of estrogen is typically low-grade endometrioid endometrial carcinoma (EEC) and has a favorable outcome. In contrast, type II EC (10-20\%) is usually non-endometrioid endometrial carcinoma (NEEC) (serous carcinoma, clear cell carcinoma and malignant mixed mullerian tumor) with high-grade tumors and poor prognosis. In pre-

\footnotetext{
* Corresponding author.

E-mail address: t-wata@fmu.ac.jp (T. Watanabe).
} 
vious reports, frequent somatic mutations of PTEN, CTNNB1, PIK3CA, ARID1A and KRAS in type I EC, as well as those of TP53 genes in type II EC, have been identified using whole exome and genome sequencing analyses [6,7]. Although several studies have reported associations between each somatic mutation and the clinicopathological characteristics of EC [8-10], few studies have focused on a comprehensive somatic mutation analysis [6]. More recently, plasma circulating tumor DNA (ctDNA) has been reported to be effective in early diagnosis and therapeutic monitoring in human cancers [11]. As blood samples are easy to obtain multiple times on demand in a non-invasive method compared with standard tumor biopsy, somatic mutations become increasingly important as clinically useful biomarkers. In the present study, we performed a comprehensive mutational profile in EC patients using a cancer panel, and examined whether somatic mutation status is associated with risk factors or survival.

\section{Materials and methods}

\section{Clinical samples}

A total of 100 surgical specimens were obtained from EC patients who had undergone surgery at Fukushima Medical University Hospital between August 2013 and December 2017. The clinicopathological data for age, staging (FIGO 2008), histology, tumor grade (EEC), MI, VI, LNM, and recurrence were collected by operative reports, clinical notes and pathological reports. Histology was divided into EEC and NEEC, and adjuvant therapy was determined according to the physician's treatment strategy. The study design was approved by the ethics committee of Fukushima Medical University (No. 1953), and informed consent was obtained from all participants. All analyses were performed in accordance with the relevant guidelines and regulations.

\section{DNA extraction}

Isolation and purification of genomic DNAs after extraction of RNAs from 100 frozen tumor samples were performed using ISOGEN reagent (Nippongene, Tokyo, Japan), according to the manufacturer's instructions. The concentration and quality of each DNA sample were assessed using NanoDrop One (ThermoFisher Scientific, Waltham, MA, USA).

\section{Somatic mutation detection}

The Ion Ampliseq Cancer Hotspot Panel v2 on the Ion Torrent platform was used to detect 2790 mutations in 50 oncogenes and tumor suppressor genes [12,13]. In brief, $10 \mathrm{ng}$ of genomic DNAs extracted from 100 frozen tumor samples were used to construct barcoded DNA libraries utilizing an Ion Ampliseq Library Kit 2.0 (Thermo Fisher Scientific). The obtained libraries were optimized using an Ion Library Equalizer Kit (Thermo Fisher Scientific), and then sequenced using an Ion Personal Genome Machine or Ion S5XL platform (Thermo Fisher Scientific). The sequencing reads were aligned to the reference genome build hg19, GRCh37, and converted into BAM files using Ion Torrent Suite software (Thermo Fisher Scientific). Sequence variants were then called using Ion Reporter 5.0 (Thermo Fisher Scientific), according to the manufacturer's instructions. The average sequencing depth reached at least 1500 -fold per sample.

\section{Statistical analysis}

The associations between somatic mutations and categorical variables, as well as between mutation rate or frequency and categorical variables, were evaluated using the chi-squared test or Mann-Whitney $U$ Test. OS was evaluated as clinical outcomes. Survival distributions were calculated according to the Kaplan-Meier method, and statistical significance was determined using the log-rank test. Values of $P<0.05$ were considered statistically significant. Statistical analysis of data was performed using SPSS version 25 software (SPSS, Inc., Chicago, IL, USA).
Table 1

Clinicopathological characteristics of patients with endometrial cancer.

\begin{tabular}{|c|c|c|}
\hline Characteristics & No. of cases & $(\%)$ \\
\hline \multicolumn{3}{|c|}{ Age at diagnosis (years) } \\
\hline$<60$ & 45 & $(45)$ \\
\hline$\geq 60$ & 55 & (55) \\
\hline \multicolumn{3}{|l|}{ Stage } \\
\hline I & 61 & (61) \\
\hline II & 9 & (9) \\
\hline III & 14 & (14) \\
\hline IV & 16 & (16) \\
\hline \multicolumn{3}{|l|}{ Histology } \\
\hline EEC & 82 & $(82)$ \\
\hline NEEC & 18 & (18) \\
\hline \multicolumn{3}{|l|}{ Grade } \\
\hline G1 & 54 & $(65.9)$ \\
\hline G2 & 16 & (19.5) \\
\hline G3 & 12 & (14.6) \\
\hline \multicolumn{3}{|c|}{ Myometrial invasion } \\
\hline$>1 / 2$ & 54 & (54) \\
\hline$\leq 1 / 2$ & 46 & (46) \\
\hline \multicolumn{3}{|c|}{ Vascular invasion } \\
\hline No & 69 & (69) \\
\hline Yes & 31 & (31) \\
\hline \multicolumn{3}{|c|}{ Lymph node metastasis } \\
\hline No & 84 & (84) \\
\hline Yes & 16 & (16) \\
\hline \multicolumn{3}{|l|}{ Recurrence } \\
\hline No & 78 & (78) \\
\hline Yes & 22 & $(22)$ \\
\hline
\end{tabular}

\section{Results}

\section{Clinicopathological characteristics}

The clinicopathological characteristics of the patients are shown in Table 1. A total of 100 EC patients who pervious undergone surgery were enrolled in this study. The median age at diagnosis was 62.5 years (range, 32-89 years), and 62 (62\%) of the patients with high recurrence risk or advanced stage underwent platinum-based chemotherapy postoperatively. Among the 100 tumors, 82 were EEC and 18 were NEEC (nine serous, six clear cell, two undifferentiated and one mixed carcinoma). After a median follow-up of 37 months (range, 1-76 months), $78(78 \%)$ patients were alive without clinical evidence of tumor. Recurrence was identified during the follow-up period in $22(22 \%)$ patients: six $(6 \%)$ patients were alive with disease; sixteen $(16 \%)$ patients died due to their tumor between postoperative months 1 and 42 (median, 7.5 months).

\section{Spectrum and frequency of mutations in EC}

In order to explore the spectrum and frequency of mutations in EC, 100 frozen tumors were analyzed. Fig. 1 shows a summary of somatically altered genes that are recurrently mutated. Validated mutations were detected in 91 of the 100 tumors (91\%), and 77 tumors (77\%) harbored concurrent mutations in two or more genes. Of 50 tumor-related genes, 38 were observed in EC tumors in the current study. Mutations were most frequently detected in PTEN (57\%), PIK3CA (51\%) and TP53 (30\%) (Fig. 1). KRAS, CTNNB1, FBFR2, FBXW7 and retinoblastoma (RB) gene mutations were relatively frequent, with respective occurrence rates of $23 \%, 21 \%, 13 \% .10 \%$ and $9 \%$, followed by APC (6\%); SMARCB1 (5\%); AKT1, ATM, BRAF, ERBB2 and MET, (4\%); ERBB4, GNAS, KIT, SMAD4, SMO and STK11 (3\%); ABL, FGFR3, HNF1A, KDR and NRAS (2\%); and EGFR, CDH1, CDKN2A, EZH2, EGFR1, GNAQ IDH1, JAK2, MLH1, MPL, NOTCH1 and VHL (1\%) (Fig. 1). A total of 284 (mean, 2.84) mutations were detected: 212 (74.6\%) missense mutations; 39 (13.7\%) nonsense mutations; 26 (9.2\%) frameshift indels; and seven (2.5\%) non-frameshift indels (Fig. 1). 


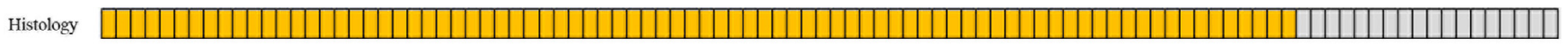

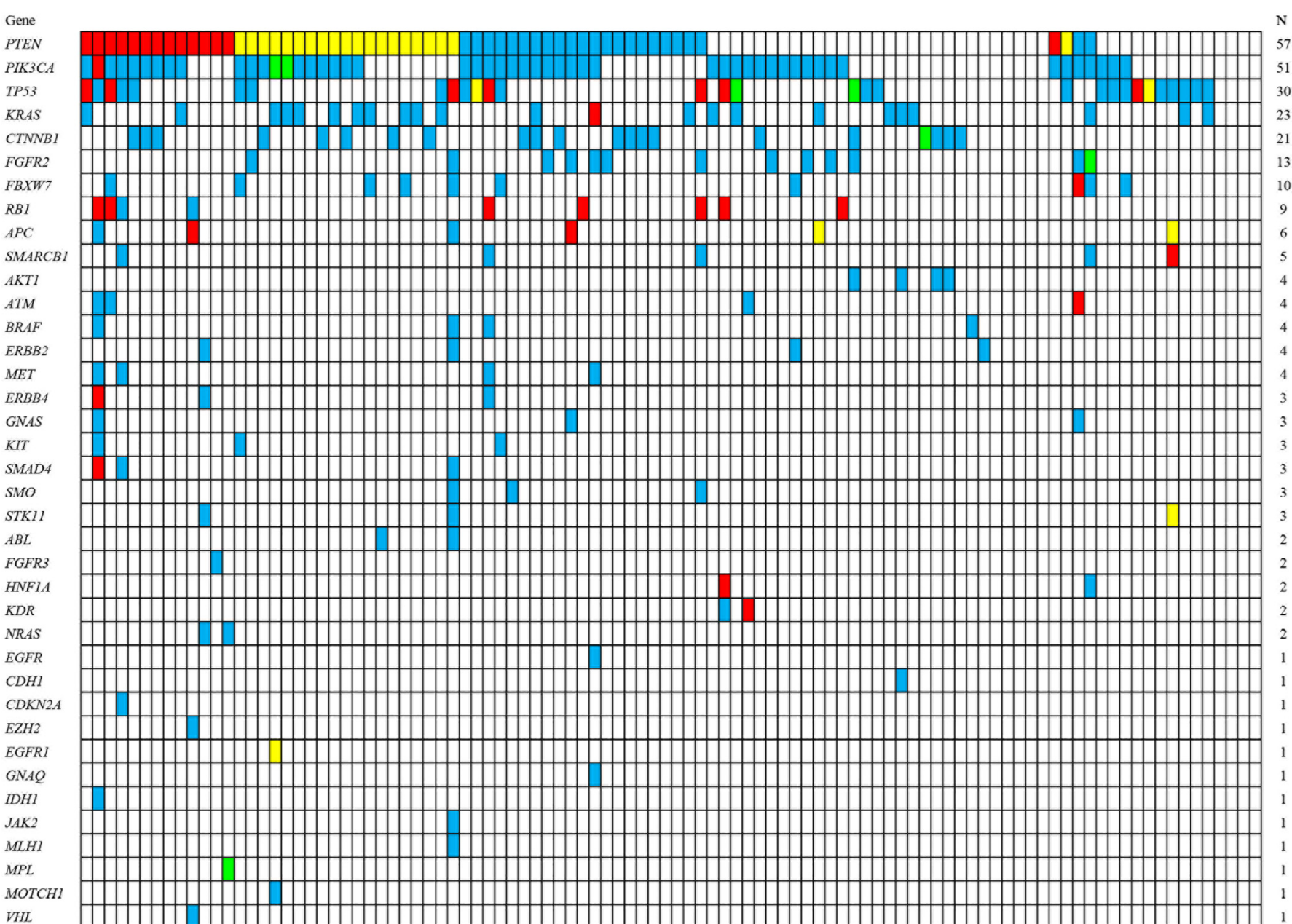

Histology $\square$ Endometrioid carcinoma $\square$ Non-Endometrioid carcinoma
Mutations $\square$ Missense $\square$ Nonsense $\square$ Frameshift IndDel $\square$ Non-Frameshift InDel

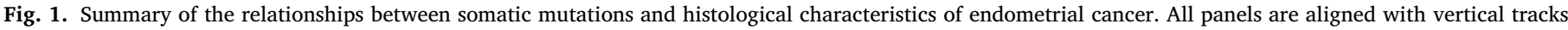
representing 100 individuals.

We examined the differences in mutation rates between EEC and NEEC using a chi-squared test. The mutation rate was significantly higher in the EEC $(93.9 \%, 77 / 82)$ than in the NEEC $(77.7 \%, 14 / 18)$ $(P=0.03)$ (data not shown). The association between mutation frequencies and clinicopathological features was investigated using the Mann-Whitney $U$ Test. Table S1 shows the mean mutation frequency with standard deviation and P-value. Age, tumor grade and histology were significantly associated with mutation frequencies (Fig. 2a-c). On the other hand, there were no significant associations between stage, MI, VI, LNM, or recurrence and mutation frequencies (Supplementary Table 1).

Relationships between mutation status and clinicopathological characteristics

Table 2 shows a summary of the associations between eight most frequently mutated genes (PTEN, PIK3CA, TP53, KRAS, CTNNB1, FBFR2, $F B X W 7$ and $R B$ ) and clinicopathological characteristics in EC patients. Patients with PTEN and CTNNB1 mutations had a significantly young age $(<60)$ than those without these mutations $(P=0.003$ and $P=0.006$, respectively). The TP53 and $F B X W 7$ mutations were significantly observed in late-stage EC ( $P=0.017$ and $P=0.023$, respectively), whereas the PTEN mutation was significantly more common in early-stage EC
$(P=0.007)$. TP53 mutation was significantly more common in the NEEC tumors $(P=0.001)$, whereas the PTEN and CTNNB1 mutations were significantly more common in the EEC tumors $(P=0.001$ and $P=0.02$, respectively). TP53 mutation was significantly associated with high-grade tumor $(P=0.0001)$. FGFR2 mutation was significantly associated with in deep $(\geq 1 / 2)$ MI $(P=0.016)$. On the other hand, the association of the CTNNB1 mutation with superficial $(<1 / 2)$ MI was marginally significant $(P=0.071)$. FBXW7 mutation was significantly associated with VI $(P=0.001)$, And the association between TP53 mutation and VI was also marginally significant $(P=0.088)$. In contrast, the association between CTNNB1 mutation and non-VI was marginally significant $(P=0.064)$, and FBXW7 mutation was significantly associated with $\operatorname{LNM}(P=0.029)$. The association of TP53 mutation with LNM was also marginally significant $(P=0.056)$. On the other hand, the association of PTEN mutation with non-LNM was also marginally significant $(P=0.08)$. TP53 mutation was significantly associated with recurrence $(P=0.004)$, whereas $P T E N$ mutation was significantly associated with non-recurrence $(P=0.001)$.

Association of mutation status with clinical survival

The relationships between the eight most frequently mutated genes (PTEN, PIK3CA, TP53, KRAS, CTNNB1, FBFR2, FBXW7 and RB1) and clinical survival were analyzed by log-rank test. The Kaplan-Meier 


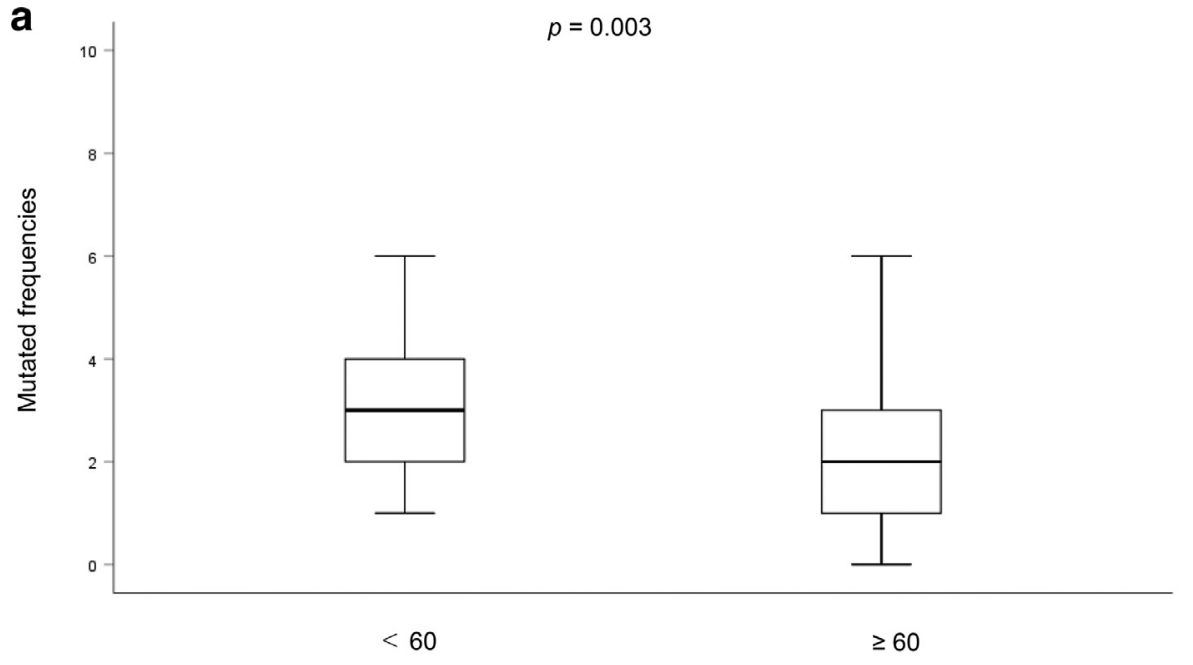

b
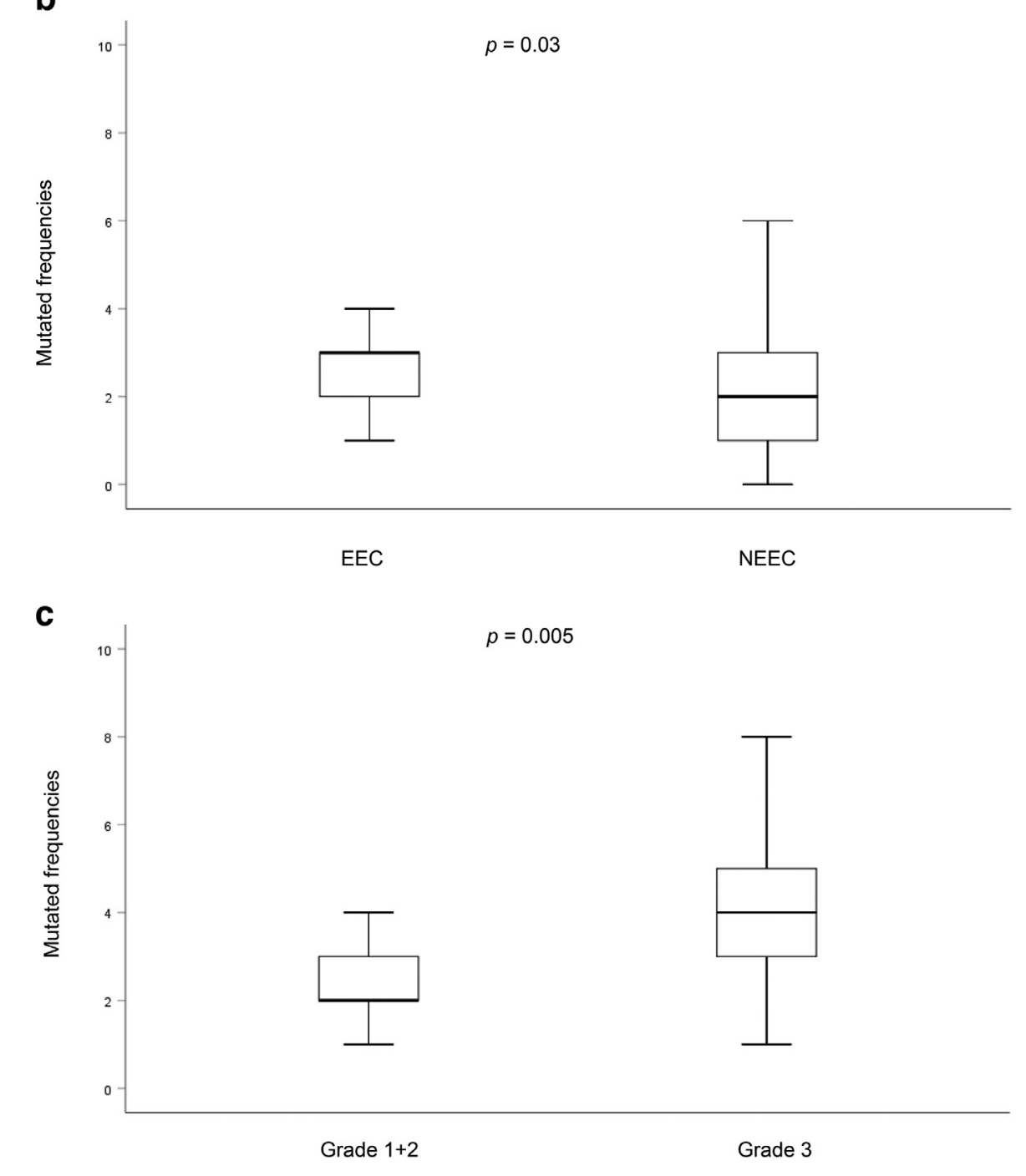

Fig. 2. Box plot showing differences in mutation frequency between (a) $<60$ and $\geq 60(P=0.003)$. (b) Grade $1+2$ and Grade $3(P=0.005)$. (c) EEC and NEEC $(P=0.03)$. EEC: endometrial endometrioid carcinoma, NEEC: non-endometrial endometrioid carcinoma curves for PTEN, TP53 and CTNNB1 mutations in the EC patients are shown in Fg. 3. The patients with PTEN and/or CTNNB1 mutations had a significantly better OS than those without these mutations $(P=0.019$ and $P=0.033$, respectively) (Fig. 3a and b), whereas those with TP53 mutation had a significantly worse OS $(P=0.001)$ (Fig. 3c). However, PIK3CA, KRAS, FBFR2, FBXW7 and RB1 mutations had no significant associations with OS (data not shown).

\section{Discussion}

Our findings showed a very high rate $(91 \%)$ of a potential oncogenic driver or drug targetable mutations in EC patients. Validated mutations have been also reported in 78-94\% of EC patients using a cancer panel $[14,15]$, and EC is known as a tumor with a high frequency of mutated genes [16]. A comprehensive genomic analysis by The Can- 
Table 2

Frequency of PTEN, PIK3CA, TP53, KRAS, CTNNB1, FGFR2, FBXW7, and RB mutations according to demographic and clinicopathological characteristics.

\begin{tabular}{|c|c|c|c|c|c|c|c|c|c|c|c|c|}
\hline $\begin{array}{l}\text { Gene } \\
\text { Characteristic }\end{array}$ & $\begin{array}{l}\text { PTEN } \\
\text { Wt } \\
n=43\end{array}$ & $\begin{array}{l}\text { Mut } \\
n=57\end{array}$ & $P$ value & $\begin{array}{l}\text { PIK3CA } \\
\mathrm{Wt} \\
n=49\end{array}$ & $\begin{array}{l}\text { Mut } \\
n=51\end{array}$ & $P$ value & $\begin{array}{l}\text { TP53 } \\
\text { Wt } \\
n=70\end{array}$ & $\begin{array}{l}\text { Mut } \\
n=30\end{array}$ & $P$ value & $\begin{array}{l}\text { KRAS } \\
\text { Wt } \\
n=77\end{array}$ & $\begin{array}{l}\text { Mut } \\
n=23\end{array}$ & $P$ value \\
\hline \multicolumn{13}{|l|}{ Age } \\
\hline$<60$ & 12 & 33 & 0.003 & 19 & 26 & 0.22 & 31 & 14 & 0.83 & 35 & 10 & 0.87 \\
\hline$\geq 60$ & 31 & 24 & & 30 & 25 & & 39 & 16 & & 42 & 13 & \\
\hline \multicolumn{13}{|l|}{ Stage } \\
\hline I/II & 24 & 46 & 0.007 & 33 & 37 & 0.57 & 54 & 16 & 0.017 & 52 & 18 & 0.32 \\
\hline III/IV & 19 & 11 & & 16 & 14 & & 16 & 14 & & 25 & 5 & \\
\hline \multicolumn{13}{|l|}{ Histology } \\
\hline EM & 29 & 53 & 0.001 & 38 & 44 & 0.26 & 63 & 19 & 0.001 & 62 & 20 & 0.48 \\
\hline Non-EM & 14 & 4 & & 11 & 7 & & 7 & 11 & & 15 & 3 & \\
\hline \multicolumn{13}{|l|}{ Grade } \\
\hline $\mathrm{G} 1 / \mathrm{G} 2$ & 26 & 44 & 0.42 & 35 & 35 & 0.11 & 59 & 11 & 0.0001 & 51 & 19 & 0.16 \\
\hline G3 & 3 & 9 & & 3 & 9 & & 4 & 8 & & 11 & 1 & \\
\hline \multicolumn{13}{|c|}{ Myometrial invasion } \\
\hline$<1 / 2$ & 22 & 32 & 0.62 & 26 & 28 & 0.85 & 33 & 13 & 0.73 & 41 & 13 & 0.78 \\
\hline$\geq 1 / 2$ & 21 & 25 & & 23 & 23 & & 37 & 17 & & 36 & 10 & \\
\hline \multicolumn{13}{|c|}{ Vascular invasion } \\
\hline No & 27 & 42 & 0.24 & 35 & 34 & 0.61 & 52 & 17 & 0.088 & 55 & 14 & 0.34 \\
\hline Yes & 16 & 15 & & 14 & 17 & & 18 & 13 & & 22 & 9 & \\
\hline \multicolumn{13}{|c|}{ Lymph node metastasis } \\
\hline No & 33 & 51 & 0.08 & 42 & 42 & 0.64 & 62 & 22 & 0.056 & 65 & 19 & 0.83 \\
\hline Yes & 10 & 6 & & 7 & 9 & & 8 & 8 & & 12 & 4 & \\
\hline \multicolumn{13}{|l|}{ Recurrence } \\
\hline No & 27 & 51 & 0.001 & 38 & 40 & 0.91 & 60 & 18 & 0.004 & 59 & 19 & 0.54 \\
\hline \multirow[t]{2}{*}{ Yes } & 16 & 6 & & 11 & 11 & & 10 & 12 & & 18 & 4 & \\
\hline & $\begin{array}{l}\text { CTNNB1 } \\
\text { Wt } \\
n=79\end{array}$ & $\begin{array}{l}\text { Mut } \\
n=21\end{array}$ & $P$ value & $\begin{array}{l}\text { FGFR2 } \\
\text { Wt } \\
n=87\end{array}$ & $\begin{array}{l}\text { Mut } \\
n=13\end{array}$ & $P$ value & $\begin{array}{l}\text { FBXW7 } \\
\text { Wt } \\
n=90\end{array}$ & $\begin{array}{l}\text { Mut } \\
n=10\end{array}$ & $P$ value & $\begin{array}{l}\mathrm{RB} \\
\mathrm{Wt} \\
n=91 \\
\end{array}$ & $\begin{array}{l}\text { Mut } \\
n=9\end{array}$ & $P$ value \\
\hline \multicolumn{13}{|l|}{ Age } \\
\hline$<60$ & 30 & 15 & 0.006 & 39 & 6 & 0.93 & 40 & 5 & 0.73 & 39 & 6 & 0.17 \\
\hline$\geq 60$ & 49 & 6 & & 48 & 7 & & 50 & 5 & & 52 & 3 & \\
\hline \multicolumn{13}{|l|}{ Stage } \\
\hline I/II & 52 & 18 & 0.077 & 63 & 7 & 0.17 & 66 & 4 & 0.023 & 64 & 6 & 0.82 \\
\hline III/IV & 27 & 3 & & 24 & 6 & & 24 & 6 & & 27 & 3 & \\
\hline \multicolumn{13}{|l|}{ Histology } \\
\hline EM & 62 & 21 & 0.02 & 71 & 11 & 0.79 & 75 & 7 & 0.29 & 73 & 9 & 0.14 \\
\hline Non-EM & 17 & 0 & & 16 & 2 & & 15 & 3 & & 18 & 0 & \\
\hline \multicolumn{13}{|l|}{ Grade } \\
\hline $\mathrm{G} 1 / \mathrm{G} 2$ & 50 & 20 & 0.14 & 61 & 9 & 0.72 & 65 & 5 & 0.28 & 64 & 6 & 0.093 \\
\hline G3 & 11 & 1 & & 10 & 2 & & 10 & 2 & & 9 & 3 & \\
\hline \multicolumn{13}{|c|}{ Myometrial invasion } \\
\hline$<1 / 2$ & 39 & 15 & 0.071 & 51 & 3 & 0.016 & 51 & 3 & 0.11 & 48 & 6 & 0.42 \\
\hline$\geq 1 / 2$ & 40 & 6 & & 36 & 10 & & 39 & 7 & & 43 & 3 & \\
\hline \multicolumn{13}{|c|}{ Vascular invasion } \\
\hline No & 51 & 18 & 0.064 & 62 & 7 & 0.21 & 67 & 2 & 0.001 & 62 & 7 & 0.55 \\
\hline Yes & 28 & 3 & & 25 & 6 & & 23 & 8 & & 29 & 2 & \\
\hline \multicolumn{13}{|c|}{ Lymph node metastasis } \\
\hline No & 65 & 19 & 0.36 & 73 & 11 & 0.94 & 78 & 6 & 0.029 & 77 & 7 & 0.59 \\
\hline Yes & 14 & 2 & & 14 & 2 & & 12 & 4 & & 14 & 2 & \\
\hline \multicolumn{13}{|l|}{ Recurrence } \\
\hline No & 59 & 19 & 0.12 & 68 & 11 & 0.55 & 72 & 6 & 0.14 & 71 & 7 & 0.91 \\
\hline Yes & 20 & 2 & & 20 & 2 & & 18 & 4 & & 20 & 2 & \\
\hline
\end{tabular}

Significant $P$ values $(P<0.05)$ were shown in bold style.

cer Genome Atlas (TCGA) resulted in the molecular classification of EC into four distinct subgroups [6]: the DNA polymerase epsilon (POLE) group, which has an exceedingly high mutation rate in conjunction with somatic mutations in the exonuclease domain of POLE; the microsatellite instability (MSI) group, which has a high mutation rate and is a hallmark of a defective mismatch repair system because of hypermethylation of the MLH1 promoter or a germline mutation in one of the mismatch repair genes; the copy number (CN)-low group, which has a low mutation rate and most of the microsatellite stable EEC; and the $\mathrm{CN}$-high group, which has a low mutation rate and consists serous carcinoma with extensive high-level $\mathrm{CN}$ alterations and frequent somatic mutations in TP53. POLE, MSI and CN-low are almost exclusively of endometrioid morphology, whereas serous carcinoma contained in NEEC is almost exclusively in the CN-high group. POLE and MSI tumors have high mutation frequencies, which are approximately 100 -fold and sixfold greater, respectively, than those seen in $\mathrm{CN}$-low or CN-high tumors. POLE-mutated tumors are frequently of high-grade EEC. In the present study, the mutated frequencies were significantly higher in high-grade EEC tumors than in the low-grade EEC tumors. Moreover, the EEC tumors had a significantly higher mutation frequency than the NEEC tumors. Although we did not examine integrated POLE mutation, or perform MSI assay or CN analysis, our findings were consistent with those of TCGA.

We here investigated the associations between the eight most frequently mutated genes and the prognostic risk factors for survival in EC patients. PTEN is identified as a tumor suppressor that is mutated in a 
a

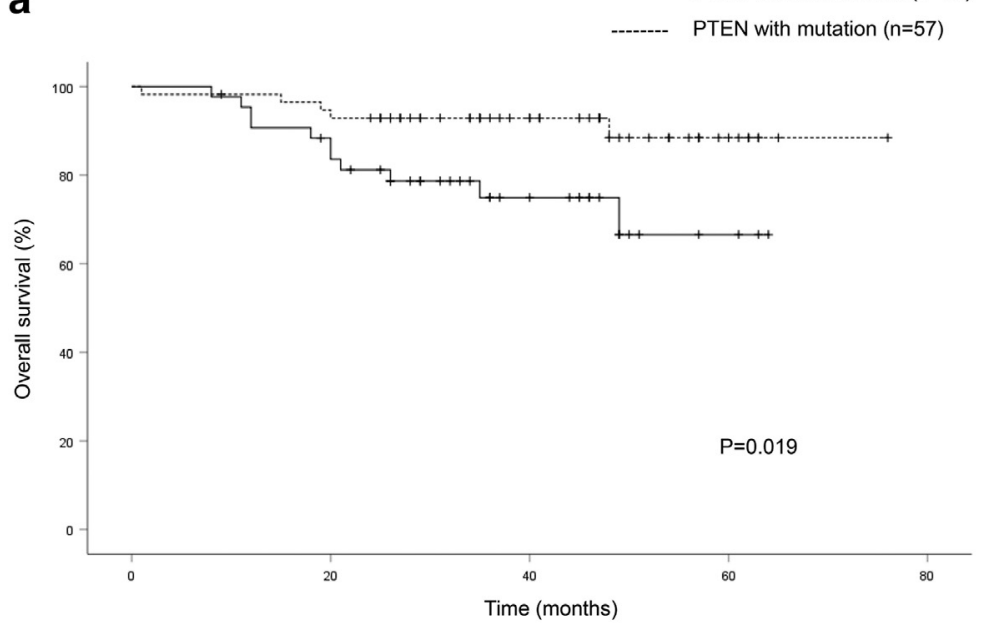

b

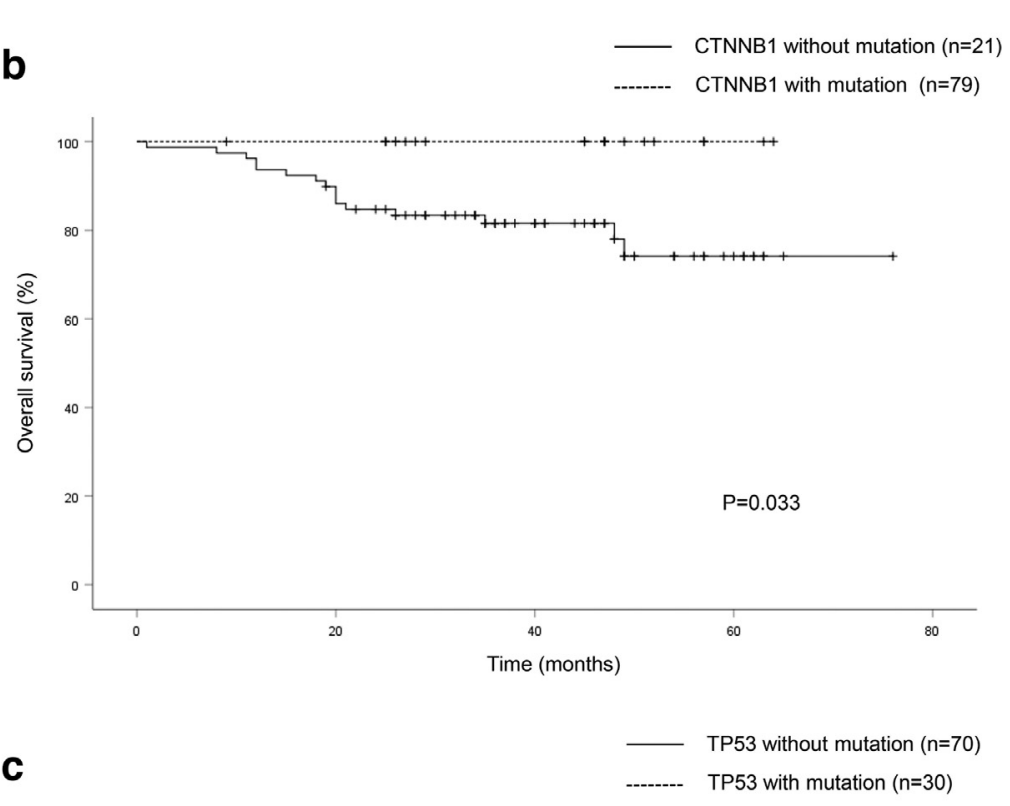

C
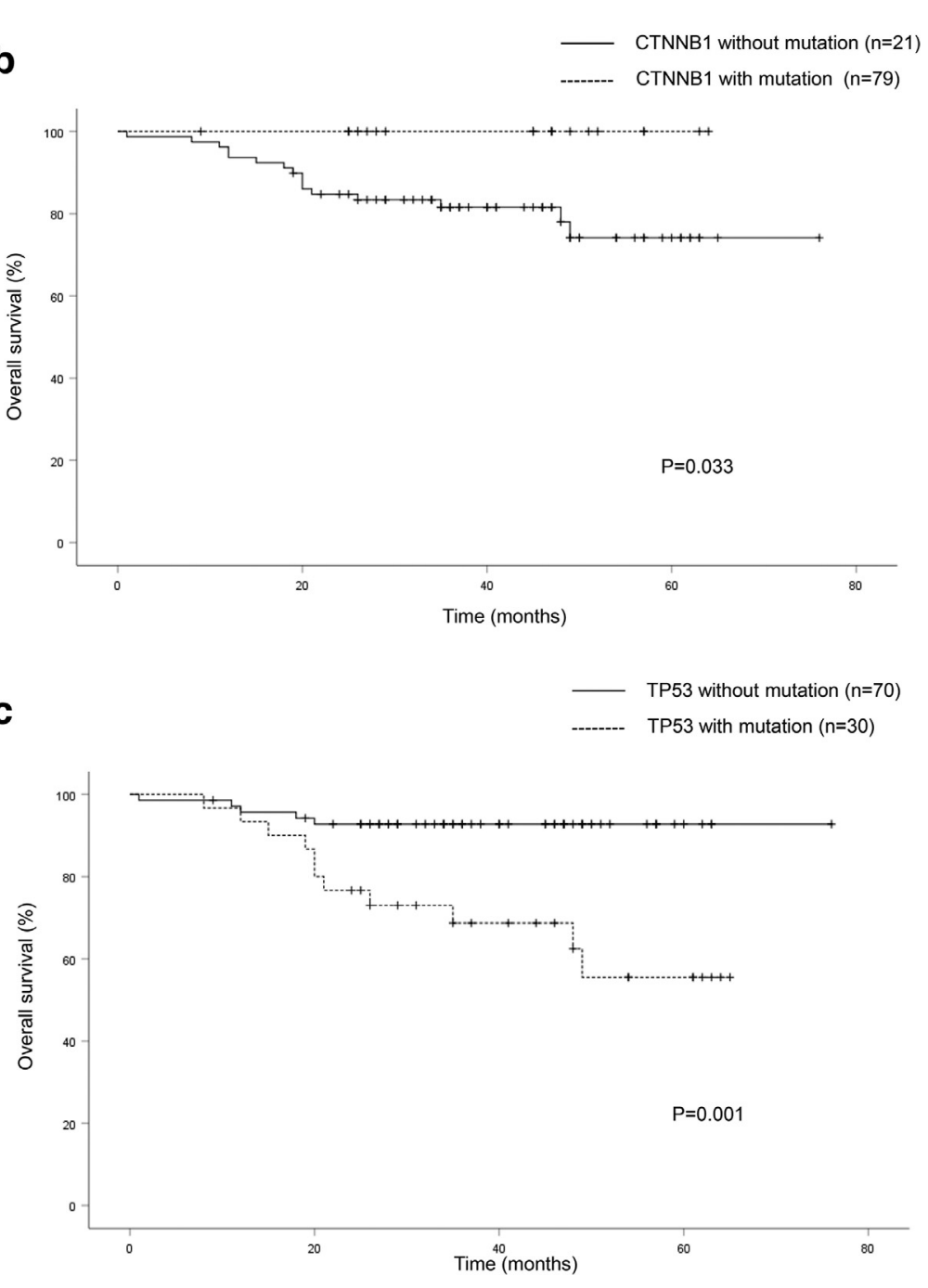

Fig. 3. The Kaplan-Meier curves of overall survival in patients with endometrial cancer. (a) Patients with and without PTEN mutations $(P=0.019)$. (b) Patients with and without CTNNB1 mutations $(P=0.033)$. (c) Patients with and without TP53 mutations $(P=0.001)$. 
PIK3CA is one of the most commonly mutated genes in solid cancers, and the majority of PIK3CA mutations cluster in hotspot regions in exon 9 (the helical domain) and exon 20 (the kinase domain) [22]. The PIK3CA mutation has been frequently detected in not only EEC but also NEEC $[23,24]$ as in the present study. As for survival, no significant association has been reported between PIK3CA mutations and diseasefree survival in $94 \mathrm{EC}$ patients [10]. On the other hand, associations have been reported between PIK3CA missense mutation and unfavorable outcome in grade 3 EEC tumors, and between PIK3CA exon 9 mutations and reduced survival in EC patients $[25,26]$. In the current study, there were no significant associations between the PIK3CA mutation and any clinicopathological features or survival. A consensus on the mechanism by which PIK3CA affects EC prognosis has yet to be achieved. Evaluation of the relationship between PIK3CA mutation and risk factors requires individualization with large samples.

Tumor suppressor $\mathrm{p} 53$ plays an important role in the preservation of genomic stability from various damages through the regulation of cellcycle checkpoints, DNA repair, senescence, and apoptosis; furthermore, TP53 is one of the most frequent alterations in human cancer. The frequency of somatic mutation in TP53 was 30\% in our study. In particular, TP53 mutations in the EEC tumors were significantly fewer than those in the NEEC tumors, and the proportion was similar to that of a previous study [27]. In addition, the associations of TP53 mutation with late-stage, high grade, and poor OS were statistically significant. Furthermore, TP53 mutations had marginally significant associations with LNM. The CN-high category represents serous-like EC, and mostly incorporates TP53 mutation, indicating a poor prognosis [6]. These results suggest that somatic mutation in TP53 may be a good predictive biomarker for poor prognosis in EC patients, and has the opposite characteristic of PTEN mutation.

The KRAS proto-oncogene regulates cell division as a result of its ability to relay external signals to the cell nucleus. Activating mutations in the KRAS gene promotes down-regulation of MAPK or PI3K/AKT, which further results in excessive cell proliferation and subsequent carcinogenesis. KRAS mutation is present in up to $25 \%$ of all human tumors, and this is one of the most frequently activated oncogenes [28]. KRAS mutations in EC have been mostly associated with type I estrogendependent EC, and their frequency is estimated around 10-30\% [2931]. Although KRAS mutation was also found in $24 \%$ (20/82) of the EEC tumors, there was no significant difference between EEC and NEEC in the present study. KRAS mutation is a relatively common event in endometrial carcinogenesis, but its prognostic value is limited. Sideris et al reported that a consensus on the exact way that KRAS overall affects EC prognosis has yet to be achieved [32]. In the present study, no significant differences between KRAS mutation and OS was observed, so the prognostic significance of KRAS mutation remains controversial.

CTNNB1 is a gene involved in the Wnt signaling pathway, which regulates cell growth, motility and differentiation. CTNNB1 mutation is the activation of the Wnt signaling pathway, and has been specifically shown to be associated with carcinogenesis in many types of tumors [33]. Since CTNNB1 mutations seemingly occur in atypical endometrial hyperplasia, CTTNB1 mutation plays a critical role in the initiation and early progression of EEC tumors [34]. Other studies reported an increase in CTNNB1 mutations in EEC compared to NEEC [35,36], and no CTNNB1 mutation was observed in the NEEC tumors in the present study. CTNNB1 mutation was detected in $25.6 \%(21 / 82)$ of the EEC tumors, which was similar to the result of previous reports [35-37]. We here demonstrated that CTNNB1 mutation was significantly associated with endometrioid subtype and favorable OS, and was borderline significantly associated with young age, early-stage, superficial MI and VI. The clinicopathological features of CTNNB1 mutations were similar to those of PTEN mutations; 90\% (19/21) of tumors with CTNNB1 mutation also had PTEN mutation. These data suggest that CTNNB1 mutation may be a favorable prognostic biomarker in addition to PTEN mutation. However, recent studies have described significant associations between CTNNB1 mutations and poor outcome among low-risk EEC patients, sug- gesting prognostic significance of CTNNB1 mutation [37-40]; thus, the clinical significance of CTNNB1 mutation among low-risk EEC tumors needs to be carefully investigated.

FGFR2 is one of the receptors for fibroblast growth factor, and has been shown to be activated in a number of cancers through a variety of mechanisms, including gene amplification, translocations, and point mutations [41]. Some studies subsequently reported a FGFR2 mutation frequency of $10-16 \%$ in EC tumors $[36,42,43]$, and said frequency in the current study was $13 \%$. In the present study, there was a significant association between FGFR2 mutation and deep MI. To the best of our knowledge, these findings have not yet been reported. Jeske et al. suggested that clinical trials testing the efficacy of FGFR inhibitors in the adjuvant setting to prevent recurrence and death are warranted because FGFR2 mutations are associated with poor outcomes in EEC [43]. Although we did not investigate the relationships between FGFR2 mutations and OS, FGFR2 mutations may contribute to a poor prognosis for EC with MI.

FBXW7 is a critical tumor suppressor that regulates proteasomemediated degradation of various oncoproteins, such as cyclin E, c-Myc, Mcl-1, mTOR, Jun, Notch and AURKA in human cancer [44]. FBXW7 mutations have been reported in several types of human cancers and found in $2.54 \%$ across all human tumors, according to COSMIC database meta-analyses [45]. Previous studies subsequently reported an FBXW7 mutation frequency of $2-16 \%$ in EC tumors $[30,46]$, and said frequency in the current study was $10 \%$. In particular, FBXW7 mutation has been observed in $20-30 \%$ of cases of serous EC $[6,23,24]$ and was also found in $30 \%(3 / 10)$ in the present study (data not shown). The results of the present study showed that FBXW7 mutation associated with latestage, VI and LNM. In particular, the relationship between FBXW7 mutation and VI in EC was a novel finding. Loss of FBXW7 function caused by FBXW7 mutation resulted in high Brg1 expression, and was consequently associated with VI, LNM and distant metastasis in gastric cancer [47]. Although there was no significant association between FBXW7 mutation and $O S$ in the current study, FBXW7 mutation may be a predictive biomarker for poor prognosis.

The $R B 1$ gene, located on chromosome 13, is a well-known tumor suppressor gene that was discovered in genetic studies of hereditary retinoblastoma [48]. Defects in this gene are a cause of childhood cancer, bladder cancer, and osteogenic sarcoma. Loss of heterozygosity ( $\mathrm{LOH}$ ) was reported in $18 \%$ of $R B$ genes in EC and pRB downregulation was consistent with $\mathrm{LOH}$ [49]. Although $R B$ mutation was detected in $9 \%$ of EC patients, no significant association was observed between clinicopathological features or survival and $R B$ mutation. Further studies are needed with larger study populations to evaluate whether the inactivation of $R B 1$ is a useful biomarker.

However, the present study must be interpreted with caution, and a few limitations should be kept in mind. Said limitations include the small sample size, heterogeneity of histology or treatment within the cohort, and short follow-up period as they may have affected the associations between the somatic mutations and clinical outcomes. A subgroup analysis using a larger sample size would likely provide more definitive results. Another limitation is that our analysis relied on sequencing with a cancer hotspot panel, and only explained a portion of the total genetic alterations. The NGS platform used in this study detected only single nucleotide variants, and thus it was impossible to detect copy number variants and MSI status. Therefore, a large number of samples with longterm follow-up need to be prospectively analyzed using whole exome or genome sequencing that can detect single number variants, copy number variants and MSI status. Furthermore, other novel genetic or epigenetic alterations should be explored as well.

In summary, we here demonstrated that our comprehensive NGS analysis using a cancer panel was feasible for mutational profiling of EC tumors. Although whole exome and genome sequencing are useful methods for detecting somatic mutations in human cancers, they are difficult to perform in clinical practice because of problems regarding scalability and time- and cost-effectiveness. Therefore, it is necessary to provide a 
reduced set of the most informative diagnostic and prognostic clinical markers. Although the mutated gene significance for clinical outcomes in EC patients was similar to that found in previous work, new findings, the association between FGFR2 mutation and deep MI and that between FBXW7 mutation and VI, were revealed. The current study suggests that EC patients can benefit from molecular profiling with predictable prognostic factors and select systemic adjuvant chemotherapy in combination with specific targeted therapies. We believe that this work will be useful for understanding and evaluating the molecular characteristics of EC patients, and may lead to the establishment of novel treatment strategies that improve the survival of patients with EC in the future.

\section{Authorship contribution statement}

Takafumi Watanabe: Conceptualization, Methodology, Investigation, Writing - Original Draft. Hideaki Nanamiya, Daisuke Tanaka, Takao Isogai and Jun-ichi Imai: Data curation, Formal analysis. Manabu Kojima, Shinji Nomura, Shigenori Furukawa and Shu Soeda: Resources, Investigation. Shinya Watanabe: Project administration, Funding acquisition. Keiya Fujimori: Supervision Writing - Review \& Editing.

\section{Ethics approval and consent to participate}

The study protocol was approved by the Ethics Committee of Fukushima Medical University School of Medicine (No. 1953), and informed consent was obtained from all patients. All participants signed informed consent forms.

\section{Declaration of Competing Interest}

The authors declare that they have no competing interests.

\section{Funding}

The present study was supported by grants for Translational Research Programs from Fukushima Prefecture.

\section{Supplementary materials}

Supplementary material associated with this article can be found, in the online version, at doi:10.1016/j.tranon.2021.101010.

\section{References}

[1] J Ferlay, M Colombet, I Soerjomataram, C Mathers, DM Parkin, M Piñeros, et al., Estimating the global cancer incidence and mortality in 2018: GLOBOCAN sources and methods, Int. J. Cancer 144 (2019) 1941-1953.

[2] SA Sohaib, SL Houghton, R Meroni, AG Rockall, P Blake, RH Reznek, Recurrent endometrial cancer: patterns of recurrent disease and assessment of prognosis, Clin. Radiol. 62 (2007) 28-34.

[3] S Pecorelli, Revised FIGO staging for carcinoma of the vulva, cervix, and endometrium, Int. J. Gynaecol. Obstet. 105 (2009) 103-104.

[4] HM Werner, HB. Salvesen, Current status of molecular biomarkers in endometrial cancer, Curr. Oncol. Rep. 16 (2014) 403.

[5] MR Stratton, PJ Campbell, PA Futreal, The cancer genome, Nature 458 (2009) 719-724.

[6] C Kandoth, N Schultz, AD Cherniack, R Akbani, Y Liu, H Shen, et al., Integrated genomic characterization of endometrial carcinoma, Nature 497 (2013) 67-73.

[7] RA Brooks, GF Fleming, RR Lastra, NK Lee, JW Moroney, CH Son, et al., Current recommendations and recent progress in endometrial cancer, CA Cancer J. Clin. 258 (2019) 258-279.

[8] JI Risinger, K Hayes, GL Maxwell, ME Carney, RK Dodge, JC Barrett, et al., PTEN mutation in endometrial cancers is associated with favorable clinical and pathologic characteristics, Clin. Cancer Res. 4 (1998) 3005-3010.

[9] EJ Lee, TJ Kim, DS Kim, CH Choi, JW Lee, JH Lee, et al., p53 alteration independently predicts poor outcomes in patients with endometrial cancer: a clinicopathologic study of 131 cases and literature review, Gynecol. Oncol. 116 (2010) 533-538.

[10] Y Dong, X Yang, O Wong, X Zhang, Y Liang, Y Zhang, et al., PIK3CA mutations in endometrial carcinomas in Chinese women: phosphatidylinositol 3'-kinase pathway alterations might be associated with favorable prognosis, Hum. Pathol. 43 (2012) 1197-1205.

[11] ML Cheng, E Pectasides, GJ Hanna, HA Parsons, AD Choudhury, GR Oxnard, Circulating tumor DNA in advanced solid tumors: clinical relevance and future directions, CA Cancer J. Clin. 9 (2020), doi:10.3322/caac.21650.
[12] A Hodgson, Y Amemiya, A Seth, M Cesari, B Djordjevic, C Parra-Herran, Genomic abnormalities in invasive endocervical adenocarcinoma correlate with pattern of invasion: biologic and clinical implications, Mod. Pathol. 30 (2017) 1633-1641.

[13] MV Nemtsova, AI Kalinkin, EB Kuznetsova, IV Bure, EA Alekseeva, II Bykov, et al., Clinical relevance of somatic mutations in main driver genes detected in gastric cancer patients by next-generation DNA sequencing, Sci. Rep. 10 (2020) 504.

[14] GM Boland, SA Piha-Paul, V Subbiah, M Routbort, SM Herbrich, K Baggerly, et al., Clinical next generation sequencing to identify actionable aberrations in a phase I program, Oncotarget 6 (2015) 20099-20110.

[15] M Wang, W Fan, M Ye, C Tian, L Zhao, J Wang, et al., Molecular profiles and tumor mutational burden analysis in Chinese patients with gynecologic cancers, Sci. Rep. 8 (2018) 8990.

[16] MH Bailey, C Tokheim, E Porta-Pardo, S Sengupta, D Bertrand, A Weerasinghe, et al., Comprehensive characterization of cancer driver genes and mutations, Cell 173 (2018) 371-385.

[17] MK McConechy, J Ding, MC Cheang, K Wiegand, J Senz, A Tone, Y, et al., Use of mutation profiles to refine the classification of endometrial carcinomas, J. Pathol. 228 (2012) 20-30.

[18] RL Levine, CB Cargile, MS Blazes, B van Rees, RJ Kurman, LH Ellenson, PTEN mutations and microsatellite instability in complex atypical hyperplasia, a precursor lesion to uterine endometrioid carcinoma, Cancer Res. 58 (1998) 3254-3258.

[19] GL Mutter, MC Lin, JT Fitzgerald, JB Kum, JP Baak, JA Lees, et al., Altered PTEN expression as a diagnostic marker for the earliest endometrial precancers, J. Natl. Cancer Inst. 92 (2000) 924-930.

[20] H Sun, T Enomoto, M Fujita, H Wada, K Yoshino, K Ozaki, et al., Mutational analysis of the PTEN gene in endometrial carcinoma and hyperplasia, Am. J. Clin. Pathol. 115 (2001) 32-38.

[21] HB Salvesen, I Stefansson, EI Kretzschmar, P Gruber, ND MacDonald, A Ryan, et al., Significance of PTEN alterations in endometrial carcinoma: a population-based study of mutations, promoter methylation and PTEN protein expression, Int. J. Oncol. 25 (2004) 1615-1623.

[22] G Ligresti, L Militello, LS Steelman, A Cavallaro, F Basile, F Nicoletti, et al., PIK3CA mutations in human solid tumors: role in sensitivity to various therapeutic approaches, Cell Cycle 8 (2009) 1352-1358.

[23] E Kuhn, RC Wu, B Guan, G Wu, J Zhang, Y Wang, et al., Identification of molecular pathway aberrations in uterine serous carcinoma by genome-wide analyses, J. Natl. Cancer Inst. 104 (2012) 1503-1513.

[24] M Le Gallo, AJ O'Hara, ML Rudd, ME Urick, NF Hansen, NJ O’Neil, et al., Exome sequencing of serous endometrial tumors identifies recurrent somatic mutations in chromatin-remodeling and ubiquitin ligase complex genes, Nat. Genet. 44 (2012) 1310-1315.

[25] JB McIntyre, GS Nelson, P Ghatage, D Morris, MA Duggan, CH Lee, et al., PIK3CA missense mutation is associated with unfavorable outcome in grade 3 endometrioid carcinoma but not in serous endometrial carcinoma, Gynecol. Oncol. 132 (2014) 188-193.

[26] S Mjos, HMJ Werner, E Birkeland, F Holst, A Berg, MK Halle, et al., PIK3CA exon9 mutations associate with reduced survival, and are highly concordant between matching primary tumors and metastases in endometrial cancer, Sci. Rep. 7 (2017) 10240.

[27] AM Schultheis, LG Martelotto, MR De Filippo, S Piscuglio, CK Ng, YR Hussein, et al., TP53 mutational spectrum in endometrioid and serous endometrial cancers, Int. J. Gynecol. Pathol. 35 (2016) 289-300.

[28] O Kranenburg, The KRAS oncogene: past, present, and future, Biochim. Biophys. Acta 25 (2005) 81-82

[29] D Llobet, J Pallares, A Yeramian, M Santacana, N Eritja, A Velasco, et al., Molecular pathology of endometrial carcinoma: practical aspects from the diagnostic and therapeutic viewpoints, J. Clin. Pathol. 62 (2009) 777-785.

[30] DA Garcia-Dios, D Lambrechts, L Coenegrachts, I Vandenput, A Capoen, PM Webb, et al., High-throughput interrogation of PIK3CA, PTEN, KRAS, FBXW7 and TP53 mutations in primary endometrial carcinoma, Gynecol. Oncol. 128 (2013) 327-334.

[31] K Banno, M Yanokura, M Iida, K Masuda, D Aoki, Carcinogenic mechanisms of endometrial cancer: Involvement of genetics and epigenetics, J. Obstet. Gynaecol. Res. 40 (2014) 1957-1967.

[32] M Sideris, EI Emin, Z Abdullah, J Hanrahan, KM Stefatou, V Sevas, et al., The role of KRAS in endometrial cancer: a mini-review, Anticancer Res. 39 (2019) 533-539.

[33] A Klaus, W Birchmeier, Wnt signaling and its impact on development and cancer, Nat. Rev. Cancer 8 (2008) 387-398.

[34] G Moreno-Bueno, D Hardisson, D Sarrio, C Sanchez, R Cassia, J Prat, et al., Abnormalities of E- and P-cadherin and catenin (beta-, gamma-catenin, and p120ctn) expression in endometrial cancer and endometrial atypical hyperplasia, J. Pathol. 199 (2003) 471-478.

[35] P Machin, L Catasus, C Pons, J Munoz, X Matias-Guiu, J Prat, CTNNB1 mutations and beta-catenin expression in endometrial carcinomas, Hum. Pathol. 33 (2002) 206-212.

[36] SA Byron, M Gartside, MA Powell, CL Wellens, F Gao, DG Mutch, et al., FGFR2 point mutations in 466 endometrioid endometrial tumors: relationship with MSI, KRAS, PIK3CA, CTNNB1 mutations and clinicopathological features, PLoS One 7 (2012) e30801.

[37] Y Liu, L Patel, GB Mills, KH Lu, AK Sood, L Ding, et al., Clinical significance of CTNNB1mutation and Wnt pathway activation in endometrioid endometrial carcinoma, J. Natl. Cancer Inst. 106 (2014) dju245.

[38] KC Kurnit, GN Kim, BM Fellman, DL Urbauer, GB Mills, W Zhang, et al., CTNNB1 (beta-catenin) mutation identifies low grade, early stage endometrial cancer patients at increased risk of recurrence, Mod. Pathol. 30 (2017) 1032-1041. 
[39] A Myers, WT Barry, MS Hirsch, U Matulonis, L Lee, Beta-catenin mutations in recurrent FIGO IA grade I endometrioid endometrial cancers, Gynecol. Oncol. 134 (2014) 426-427.

[40] E Stelloo, RA Nout, EM Osse, IJ Jürgenliemk-Schulz, JJ Jobsen, LC Lutgens, et al., Improved risk assessment by integrating molecular and clinicopathological factors in early-stage endometrial cancer-combined analysis of the PORTEC cohorts, Clin. Cancer Res. 22 (2016) 4215-4224.

[41] C Hierro, J Rodon, J Tabernero, Fibroblast growth factor (FGF) receptor/FGF inhibitors: novel targets and strategies for optimization of response of solid tumors, Semin. Oncol. 42 (2015) 801-819.

[42] PM Pollock, MG Gartside, LC Dejeza, MA Powell, MA Mallon, et al., Frequent activating FGFR2 mutations in endometrial carcinomas parallel germline mutations associated with craniosynostosis and skeletal dysplasia syndromes, Oncogene 26 (2007) 7158-7162.

[43] YW Jeske, S Ali, SA Byron, F Gao, RS Mannel, RG Ghebre, et al., FGFR2 mutations are associated with poor outcomes in endometrioid endometrial cancer: an NRG Oncology/Gynecologic Oncology Group study, Gynecol. Oncol. 145 (2017) 366-373.
[44] CH Yeh, M Bellon, C Nicot, FBXW7: a critical tumor suppressor of human cancers, Mol. Cancer 17 (2018) 115.

[45] SA Forbes, D Beare, H Boutselakis, S Bamford, N Bindal, J Tate, et al., COSMIC: somatic cancer genetics at high-resolution, Nucleic Acids Res. 45 (2017) D777-D783.

[46] CH Spruck, H Strohmaier, O Sangfelt, HM Müller, M Hubalek, E Müller-Holzner, et al., hCDC4 gene mutations in endometrial cancer, Cancer Res. 45 (2002) $4535-4539$.

[47] LY Huang, J Zhao, H Chen, L Wan, H Inuzuka, J Guo, et al., SCFFBW7-mediated degradation of Brg1 suppresses gastric cancer metastasis, Nat. Commun. 9 (2018) 3569.

[48] JL Berry, A Polski, WK Cavenee, TP Dryja, AL Murphree, BL Gallie, The RB1 story: characterization and cloning of the first tumor suppressor gene, Genes 10 (2019) 879.

[49] A Semczuk, B Marzec, A Roessner, JA Jakowicki, J Wojcierowski, R Schneider-Stock, Loss of heterozygosity of the retinoblastoma gene is correlated with the altered pRb expression in human endometrial cancer, Virchows Arch. 441 (2002) 577-583. 